\title{
A. Heck
}

\author{
Astronomical Observatory, Strasbourg
}

ABSTRACT. This paper presents a brief review of the various applications carried out with the statistical-parallax algorithm based on the principle of maximum likelihood.

\section{INTRODUCTION}

The algorithm of statistical parallaxes based on the principle of maximum likelihood and developed successively by Rigal (1958), Jung (1968, 1970) and Heck (1975a) has proven to be a reliable method for determining the absolute luminosity and kinematics of a number of stellar groups under a minimum of assumptions. For comparative methodological reviews, please refer to Heck (1976 \& 1978b).

Let us just recall here that, under its present generalized form, the $\mathrm{RJH}$ algorithm permits the calibration of a relation between individual stellar absolute magnitudes $\left(\mathrm{M}_{i}\right)$ of a given sample and a number of observable quantities $\left(Q_{i j}\right)$ of the type

$$
M_{i}=\sum_{j=1}^{n} a_{j} Q_{i j} .
$$

Obviously, if $n=1$ and $Q_{i 1}=1$ for all $i$, $a_{1}$ represents the sample mean absolute magnitude. The mean solar motion and the corresponding velocity ellipsoid are simultaneously determined. Moreover, numerical simulations provide the precision of each estimate. Stars with extreme kinematical properties (i.a. high-velocity stars) are eliminated from the sample by tests on the space velocities.

The method requires for each star the knowledge of the following data: position (not critical), proper motion components and errors, radial velocity and error, apparent magnitude, and the observable quantities $Q_{i j}$ which can be of various kinds (period, photometric indices or colors, metallicity indices, ...).

Although the algorithm can deal with raw or already corrected apparent magnitudes, it includes optionally a statistical correction for the interstellar reddening based on Parenago(1940)'s law. 373

D. S. Hayes et al. (eds.), Calibration of Fundamental Stellar Quantities, 373-375.

() 1985 by the IAU. 
This paper presents a very brief review of the applications carried out with the algorithm and which have led to a consistent set of absolute magnitudes (see Heck, 1980) for the various stellar groups considered up to now. Space is lacking in these proceedings to provide detailed synthetic tables, but we intend to do it in a forthcoming paper.

\section{APPLICATIONS OF THE RJH ALGORITHM}

The following stellar groups have been calibrated in luminosity with the RJH algorithm (roughly in chronological order):

- RR Lyrae stars (Heck, 1972, 1973a\&b, 1975b; Lakaye, 1977; Heck \& Lakaye, 1978),

- Mira variables (Foy et al., 1975),

- Strömgren's "intermediate" and "late" groups (Heck 1975c, 1977),

- late-type stars (Grenier et al., 1976a\&b, 1977),

- F-type stars (Heck, 1978a),

- Hg-Mn stars (Jaschek et al., 1980),

- Am stars (Gomez et al., 1981),

- An stars (crenier et al., 1981b),

- $\delta$ Delphini stars and $\delta$ Scuti stars (Grenier et al., 1981a),

- G5-M3 giants (Egret et al., 1982),

- F-G-K-M stars (Mikami \& Heck, 1982),

- B5-F5 dwarf and giant stars (Grenier et al., 1984).

More calibrations are considered (see i.a. Heck et al., 1981) and will be carried out every time the number of stars of a given type with all the necessary measurements will be large enough to obtain statistically significant results.

\section{REMARKS}

Another algorithm based on the maximum-likelihood principle has been described by Clube \& Jones (1971) and Clube \& Dawe (1980a\&b). Its differences from the RJH method have been discussed in Clube \& Jones $(1974)$ and Heck \& Jung (1975). A comparative study of the performance of both methods has been carried out by running simulations on a RR Lyrae star sample (Jones et al., 1980). To the best of our knowledge, the CJD algorithm has only been applied to RR Lyrae stars.

\section{REFERENCES}

Clube, S.V.M., Dawe, J.A. 1980a, Monthly Not. Roy. Astron. Soc. 190, 575 Clube, S.V.M., Dawe, J.A. 1980b, $\overline{\text { Monthly }} \overline{\text { Not. }} \overline{\text { Roy. }} \overline{\text { Astron. }} \overline{\text { Soc. }} \overline{190}, 591$ Clube, S.V.M., Jones, D.H.P. 1971, Monthly Not. Roy. Astron. Soc. 151, 231

Clube, S.V.M., Jones, D.H.P. 1974, Astron. Astrophys. 33, 153

Egret, D., Keenan, P.C., Heck, A. 1982, Astron. Astrophys. 106, 115

Foy, R., Heck, A., Mennessier, M.0. 1975, Astron. Astrophys. 43, 175

Gomez, A.E., Grenier, S., Jaschek, M., Jaschek, C., Heck, A. $\overrightarrow{1981}$, Astron. Astrophys. 93, 155 
Grenier, S., da Silva, L., Heck, A. 1976a, in Abundance Effects in Classification, ed. B. Hauck \& P.C. Keenan, (D. Reidel Publ. Co., Dordrecht), p. 215

Grenier, S., Gomez, A.E., Jaschek, C., Jaschek, M., Heck, A. 1981a, in Upper Main Sequence CP Stars, 23rd Liège Astrophys. Coll., Liège, p. 491

Grenier, S., Gomez, A.E., Jaschek, C., Jaschek, M., Heck, A. 1984 , in preparation.

Grenier, S., Jaschek, M., Gomez, A.E., Jaschek, C., Heck, A. 1981b, Astron. Astrophys. 100, 24

Grenier, S., Heck, A., Jung, J. 1976b, Astron. Astrophys. 52, 385

Grenier, S., Heck, A., Jung, J. 1977, Astron. Astrophys. Suppl. 27, 267

Heck, A. 1972, Astron. Astrophys. 21, 231

Heck, A. 1973a, Astron. Astrophys. 24, 313

Heck, A. 1973b, in Problems of Calibration of Absolute Magnitudes and Temperature of Stars, ed. B. Hauck \& B. Westerlund, (D. Reidel Publ. Co., Dordrecht), p. 21

Heck, A. 1975a, Ph. D. Thesis, Univ. Liège

Heck, A. 1975b, Astron. Astrophys. 42, 131

Heck, A. 1975c, Astron. Astrophys. 43, 111

Heck, A. 1976, Quelques méthodes de détermination de la magnitude absolue, ed. Centre de Données Stellaires, Strasbourg

Heck, A. 1977, Astron. Astrophys. 56, 235

Heck, A. 1978a, Astron. Astrophys. 66, 335

Heck, A. 1978b, Vistas in Astron. 22,221

Heck, A. 1980, Astron. Astrophys. 82, 370

Heck, A., Gomez, A.E., Grenier, S., Jaschek, C., Jaschek, M. 1981, Inf. Bull. Strasbourg Stellar Data Center 20, 34

Heck, A., Jung, J. 1975, Astron. Astrophys. 40, 323

Heck, A., Lakaye, J.M. 1978, Monthly Not. Roy. Astron. Soc. 184, 17

Jaschek, M., Jaschek, C., Grenier, S., Gomez, A.E., Heck, A. 1980, Astron. Astrophys. 81, 142

Jones, D.H.P., Heck, A., Dawe, J.A., Clube, S.V.M. 1980, Astron. Astrophys. 89,225

Jung, J. 1968, Bull. Astron. Paris, Sér. III, 3, 461

Jung, J. 1970, Astron. Astrophys. 4, 53

Lakaye, J.M. 1977, Master Thesis, Liège

Mikami, T., Heck, A. 1982, Publ. Astron. Soc. Japan 34, 529

Parenago, P.P. 1940, Astron. Zh. 17, 3

Rigal, J.L. 1958, Bull. Astron. Paris 22, 171 